\title{
Benexate hydrochloride betadex modulates nitric oxide synthesis and cytokine expression in gastric ulcers
}

\author{
JAE MIN LEE ${ }^{1 *}$, JI-YOUN LIM ${ }^{2 *}$, YOONJIN KIM ${ }^{2}$, YE JI KIM ${ }^{1}$, HYUK SOON CHOI ${ }^{1}$, EUN SUN KIM ${ }^{1}$, \\ BORA KEUM ${ }^{1}$, YEON SEOK SEO ${ }^{1}$, YOON TAE JEEN ${ }^{1}$, HONG SIK LEE ${ }^{1}$, SOON HO UM ${ }^{1}$, CHANG DUCK KIM ${ }^{1}$, \\ HO SANG RYU ${ }^{1}$, DONGGEUN SUL ${ }^{2}$, JUNGHWA HONG ${ }^{3}$ and HOON JAI CHUN ${ }^{1}$ \\ ${ }^{1}$ Division of Gastroenterology and Hepatology, Department of Internal Medicine, College of Medicine; \\ ${ }^{2}$ Graduate School of Medicine, Korea University, Seoul 02841; ${ }^{3}$ Department of Control and Instrumentation Engineering, \\ Korea University, Sejong 30019, Republic of Korea
}

Received March 19, 2015; Accepted February 11, 2016

DOI: $10.3892 / \mathrm{etm} .2016 .3384$

\begin{abstract}
The present study investigated benexate hydrochloride betadex (BHB)-mediated ulcer healing, and changes to microcirculation modulated through nitric oxide synthase (NOS) and anti-inflammatory activity. A rat model of gastric mucosal injury was established through injection of a $60 \%$ acetic acid solution into the stomach. Following ulcer induction, the rats were administered BHB orally for 5 days at doses of $0,100,300$ or $1,000 \mathrm{mg} / \mathrm{kg}$. The highest dose of BHB was also administered with or without L-NG-nitroarginine methyl ester (L-NAME). The area of gastric ulcers was determined by planimetry, and expression of cyclooxygenases (COX), cytokines and NOS in stomach tissues were measured using western blotting. Compared with the control group, gastric ulcer size was significantly decreased in the $1,000 \mathrm{mg} / \mathrm{kg}$ BHB-treated group $(\mathrm{P}<0.05)$. Administration of $\mathrm{BHB}$ led to a significant increase in endothelial (e)NOS expression $(\mathrm{P}<0.05)$. Although acetic acid co-treatment with L-NAME induced more severe mucosal damage, BHB decreased COX expression and tumor necrosis factor- $\alpha$ levels when administered with the nitric oxide inhibitor, L-NAME $(\mathrm{P}<0.05)$. BHB exhibited protective effects in a rat model of gastric ulcers, which were associated
\end{abstract}

Correspondence to: Dr Hoon Jai Chun, Division of Gastroenterology and Hepatology, Department of Internal Medicine, College of Medicine, Korea University, 73 Inchon-ro, Seoul 02841, Republic of Korea

E-mail: drchunhj@chol.com

Dr Junghwa Hong, Department of Control and Instrumentation Engineering, Korea University, 2511 Sejong-ro, Sejong 30019, Republic of Korea

E-mail: hongjh32@korea.ac.kr

*Contributed equally

Key words: benexate hydrochloride betadex, stomach, ulcer, nitric oxide, cytokine with a decrease in pro-inflammatory cytokine levels and the activation of eNOS.

\section{Introduction}

Benexate hydrochloride betadex (BHB) is used clinically as an antiulcer agent. In the gastric mucosa, BHB promotes prostaglandin synthesis, inhibits acid secretion and increases mucosal blood flow (1). The effects of BHB are proposed to be mediated by nitric oxide (NO), through inhibition of cyclooxygenases (COXs) and inflammatory cytokines. However, the mechanism by which BHB promotes mucosal angiogenesis and ulcer healing remains unclear.

NO is involved in numerous physiological functions, including healing processes (2). NO acts as a local vasodilator in the gastric mucosal microvasculature, and stimulates mucus and bicarbonate secretion in the stomach. Previous studies have suggested that the inhibition of NO synthase (NOS) significantly delays ulcer healing by reducing gastric blood flow around the ulcer (3). NO inhibitors, including L-NG-nitroarginine methyl ester (L-NAME), have an inhibitory effect on gastric ulcer healing.

COX may also be involved in gastrointestinal mucosal integrity. COX is expressed in inflammatory cells and fibroblasts of the gastric mucosa; in a previous study, COX mRNA expression increased rapidly in injured mucosa, and the level of expression was correlated with the severity of the gastric mucosal injuries (4). mRNA expression levels of certain inflammatory cytokines, including interleukin-1 $\beta$ (IL-1 $\beta$ ) and tumor necrosis factor- $\alpha$ (TNF- $\alpha)$, are also significantly increased in gastric ulcers (5).

Despite numerous studies investigating anti-gastric ulcer agents, the molecular mechanism of BHB is not fully understood. In the present study, the anti-gastric ulcer effects of BHB with respect to the modulation of NOS, COX and inflammatory cytokine expression have been investigated.

\section{Materials and methods}

Materials. BHB was purchased from Ildong Pharmaceutical Co., Ltd. (Seoul, Korea). Protein expression was detected 
using the following primary antibodies from Santa Cruz Biotechnology, Inc. (Dallas, TX, USA): Rabbit polyclonal IL-1 $\beta$ (dilution, 1:500; cat. no. sc-7884); goat polyclonal IL-6 immunoglobulin G (IgG) (dilution, 1:500; cat. no. sc-1265); goat polyclonal TNF- $\alpha$ IgG (dilution, 1:500; cat. no. sc-1351); rabbit polyclonal cyclooxygenase-1 (COX-1; dilution, 1:1,000; cat. no. sc-7950); and goat polyclonal cyclooxygenase-2 IgG (COX-2; dilution, 1:1,000; cat. no. sc-1745) (all purchased from Santa Cruz Biotechnology, Inc.). The following primary antibodies were also used: Rabbit polyclonal inducible NOS (iNOS; dilution, 1:1,000; cat. no. ab15323); rabbit monoclonal neuronal NOS (nNOS; dilution, 1:1,000; cat. no. ab76067) (both purchased from Abcam, Cambridge, UK); and rabbit monoclonal endothelial NOS (eNOS; dilution, 1:1,000; cat. no. 32027; Cell Signaling Technology, Inc., Beverly, MA, USA). Horseradish peroxidase (HRP-conjugated goat anti-mouse IgG (dilution, 1:2,000; cat. no. sc-2005), HRP-conjugated goat anti-rat IgG (dilution, 1:2,000; cat. no. sc-2006) and HRP-conjugated donkey anti-goat IgG (dilution, 1:2,000; cat. no. sc-2020) were used (all purchased from Santa Cruz Biotechnology, Inc.).

Animals. A total of 30 male Sprague-Dawley rats, weighing between 240-250 g, were purchased from Samtako Laboratory Animal Company (Osan, Korea) and housed for 1 week in the animal facility for acclimation. Constant environmental conditions were maintained with a temperature of $23 \pm 1^{\circ} \mathrm{C}$, humidity of 55\% and a 12-h light/dark cycle. Following acclimation, the rats underwent a fast for the $24 \mathrm{~h}$ prior to the experiments. The present study was approved by the Institutional Animal Care \& Use Committee of Korea University (approval number, KUIACUC-2013-181).

Study methods. Gastric ulcers were induced in all rats by direct injection of acetic acid. The rats were anesthetized using tiletamine/zolazepam $(10 \mathrm{mg} / \mathrm{kg}$; intramuscular injection; Zoetis, Inc., Florham Park, NJ, USA) and xylazine (5 mg/kg; intraperitoneal injection; Bayer AG, Leverkusen, Germany). A longitudinal incision of $2 \mathrm{~cm}$ was made in the upper abdomen. The stomach was then exposed and directly injected with $2 \mathrm{~cm}^{3}$ of $60 \%$ acetic acid solution, as described by Okabe et al (6). After $45 \mathrm{sec}$, the gastric contents were aspirated by syringe. The abdomen was sutured and oral intake of food and water was permitted following closure. Following gastric ulcer induction, the rats were randomly divided into 6 groups, with 5 rats per group. The groups were organized as follows: Control, no BHB treatment; BHB 100; 300; or $1,000 \mathrm{mg} / \mathrm{kg}$ treatment; L-NAME (Sigma-Aldrich, St. Louis, MO, USA) $70 \mathrm{mg} / \mathrm{kg}$ treatment; and L-NAME $70 \mathrm{mg} / \mathrm{kg}$ treatment with BHB at $1,000 \mathrm{mg} / \mathrm{kg}$. The drugs were dissolved in $2 \mathrm{ml} \mathrm{5 \%}$ dextrose water (DW; JW Pharmaceutical Corporation, Seoul, Korea) and administered orally once per day for 5 days. The rats in the control group were administered $2 \mathrm{ml}$ $5 \%$ DW without BHB.

Assessment of the gastric lesions. A total of 5 days after the induction of gastric ulcers, the rats were sacrificed using $\mathrm{CO}_{2}$. The stomachs were dissected, gently incised along the longer curvature, opened and rinsed with phosphate-buffered saline (PBS) to remove the gastric contents. The gastric mucosa lesions were macroscopically examined with a magnifier using a metric measurement scale. The areas of the ulcerous lesions were measured in $\mathrm{mm}^{2}$ using the lesion index (7).

Western blot analysis. The expression levels of COXs (COX-1 and COX-2), cytokines (IL-1 $\beta$, IL-6 and TNF- $\alpha$ ) and NOS (nNOS, eNOS and iNOS) were measured using western blot analysis. The gastric tissues were frozen using liquid nitrogen and stored at $-80^{\circ} \mathrm{C}$. Samples were pulverized by a mortar and pestle, then mixed with radioimmunoprecipitation assay buffer (a lysis buffer) and centrifuged at 14,200 x g for $15 \mathrm{~min}$. The supernatants were collected and the protein content was determined using a Bio-Rad Protein Assay Dye Reagent Concentrate (Bio-Rad Laboratories, Inc., Hercules, CA, USA). A similar mass of total protein was loaded from each sample onto a 5-12\% sodium dodecyl sulfate gel and transferred to polyvinylidene fluoride membranes using electrophoresis. The membranes were blocked with a blocking buffer (5\% skimmed milk in PBS) for $1 \mathrm{~h}$ at room temperature, then incubated with the primary antibody. Following several washes with PBS-Tween 20 over $30 \mathrm{~min}$, the membranes were incubated with the secondary antibody specific to the primary antibody for $1 \mathrm{~h}$ at room temperature. Following several additional washes with PBS-Tween 20 over 30 min, detection was performed using an enhanced chemiluminescence kit (Pierce ECL Western Blotting Substrate; Thermo Fisher Scientific, Inc., Waltham, MA, USA) and the images were analyzed using ImageJ software (National Institutes of Health, Bethesda, MD, USA). The intensity of each band was compared with that of the internal control, $\beta$-actin.

Statistical analysis. Data were processed and analyzed using SPSS, version 20.0 (IBM SPSS, Armonk, NY, USA). Statistical comparisons were performed using a Student's t-test. $\mathrm{P} \leq 0.05$ was considered to indicate a statistically significant difference.

\section{Results}

Gastric ulcers. The control group developed ulcerous lesions (Fig 1). Rats that received BHB at doses of 100, 300 and $1,000 \mathrm{mg} / \mathrm{kg}$ demonstrated reductions in mucosal injury of $7.8,10.7$ and $19.3 \%$, respectively, compared with the control (Fig. 2); the area of ulcerous lesions significantly decreased in the group treated with $1,000 \mathrm{mg} / \mathrm{kg}$ BHB (Fig. 2). L-NAME aggravated the acetic acid-induced ulcerous lesions, observed macroscopically (Fig. 1E), but the effect of L-NAME was somewhat reversed when it was administered with $1,000 \mathrm{mg} / \mathrm{kg}$ BHB (Fig. 1F). The L-NAME + BHB group exhibited significantly reduced lesion area compared with the L-NAME group ( $\mathrm{P}<0.05$; Fig. 2).

COX and cytokine levels of gastric ulcers. The expression levels of COX-1 and -2 are reported in Fig. 3. COX-2 expression was decreased in the $\mathrm{BHB}$ groups compared with the control group; however, a significant difference was only observed at $1,000 \mathrm{mg} / \mathrm{kg}(\mathrm{P}<0.05$; Fig. 3C). No significant difference in COX-1 expression was observed between the BHB treatment and control groups.

The expression levels of pro-inflammatory cytokines are reported in Fig. 4; and the expression levels of IL-1 $\beta$, IL-6 and TNF- $\alpha$ were not observed to be significantly different following $\mathrm{BHB}$ treatment. 


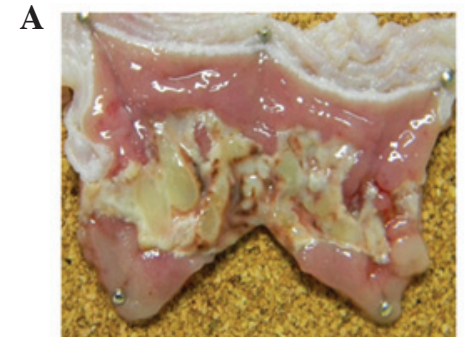

Control

D

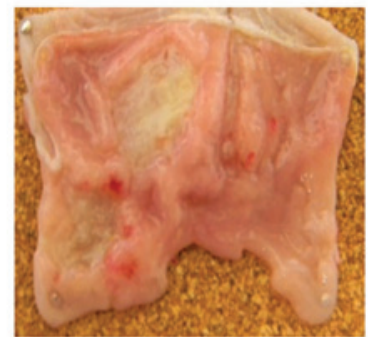

BHB 1000
B

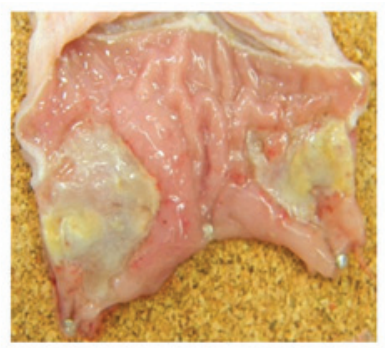

BHB 100

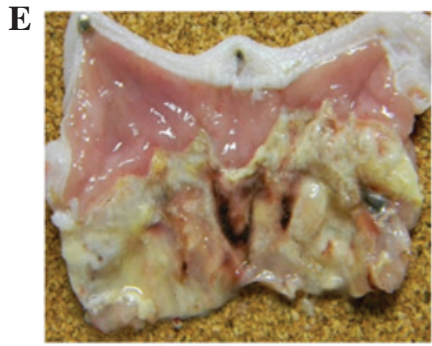

L-NAME 70

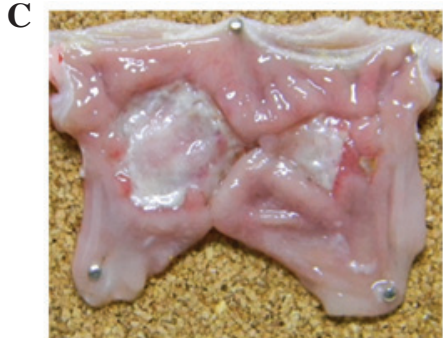

BHB 300

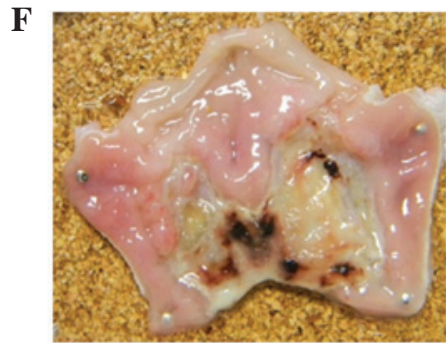

L-NAME 70 + BHB 1000

Figure 1. Macroscopic images of gastric lesions in a rat model, demonstrating acetic acid-induced mucosal damage. Treatment with (A) control, (B) 100, (C) 300 and (D) $1,000 \mathrm{mg} / \mathrm{kg} \mathrm{BHB}$, (E) $70 \mathrm{mg} / \mathrm{kg}$ L-NAME and (F) $70 \mathrm{mg} / \mathrm{kg}$ L-NAME with 1,000 mg/kg BHB. BHB, benexate hydrochloride betadex; L-NAME, L- $N G$-nitroarginine methyl ester.

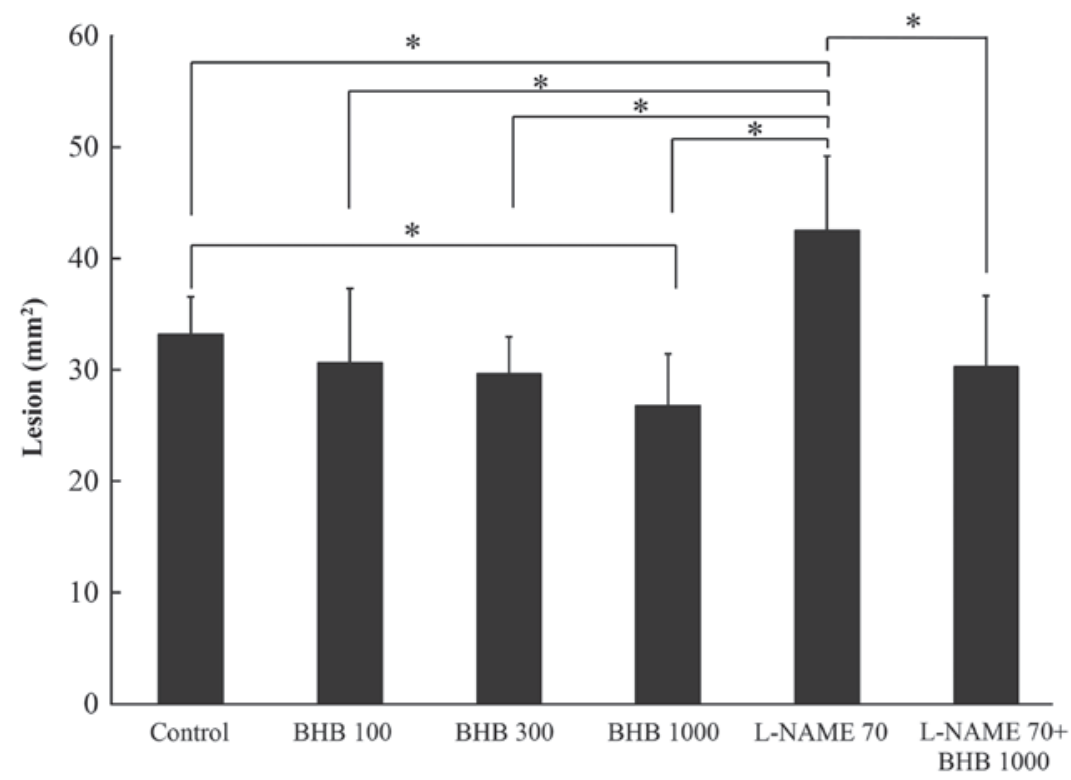

Figure 2. Pathological ulcerous lesion size in the each of the groups, following treatment with BHB and/or L-NAME, with doses provided in mg/kg. " $\mathrm{P}<0.05$, comparisons shown by brackets. BHB, benexate hydrochloride betadex; L-NAME, L- $N G$-nitroarginine methyl ester.

NOS levels in the gastric ulcers. The effect of BHB on eNOS, iNOS and nNOS protein expression was also assessed by western blot (Fig. 5A). All BHB treatments significantly increased eNOS expression compared with the control group $(\mathrm{P}<0.05$; Fig. 5B), but no significant differences were identified in the expression levels of nNOS or iNOS between the groups (Fig. 5C and D).

COX and inflammatory cytokine levels of gastric ulcers in the presence of an NO inhibitor. As presented in Fig. 6, COX-1 protein expression levels increased in rats administered L-NAME compared with the control group $(\mathrm{P}<0.05)$, and the co-treatment with BHB significantly decreased the expression of COX-1 compared with the L-NAME group $(\mathrm{P}<0.05)$. COX-2 protein expression levels increased in rats in the control group, and the administration of BHB significantly decreased COX-2 protein expression in both groups, with or without L-NAME $(\mathrm{P}<0.05)$. As presented in Fig. 7, L-NAME administration significantly increased the expression of the inflammatory cytokine TNF- $\alpha$ ( $\mathrm{P}<0.05$ vs. control group), whereas combined treatment suppressed its expression $(\mathrm{P}<0.05$ vs. L-NAME group). No significant difference was observed in the expression of IL-1 $\beta$ and IL-6 with either drug.

NOS levels of gastric ulcers in the presence of an NO inhibitor. In the presence of L-NAME, eNOS levels did not differ 

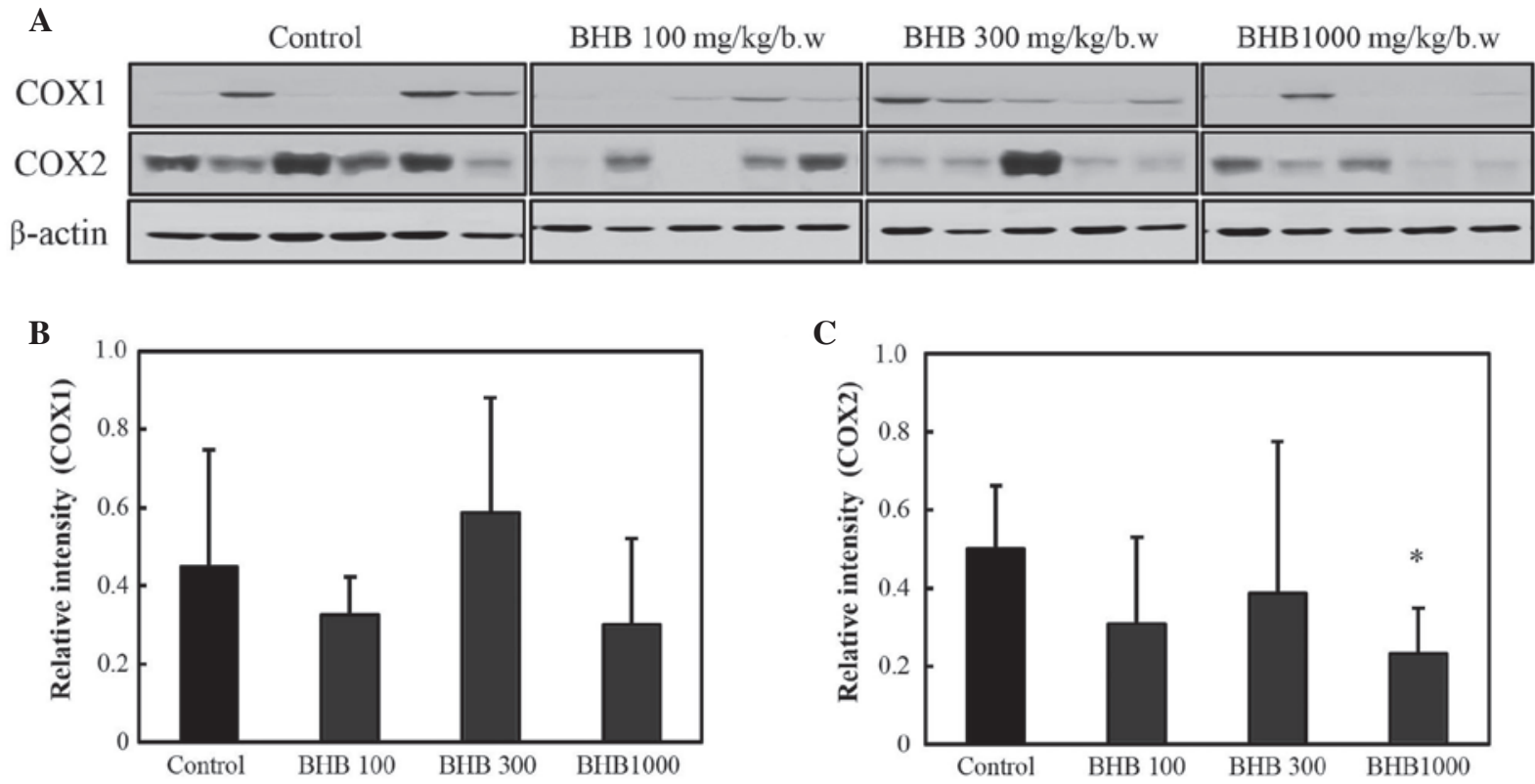

Figure 3. COX expression following treatment with BHB, with doses provided in $\mathrm{mg} / \mathrm{kg}$. (A) Western blot analysis of COX expression; (B) COX-1 and (C) COX-2 expression. ${ }^{*} \mathrm{P}<0.05$ vs. the control group. BHB, benexate hydrochloride betadex; COX, cyclooxygenase.
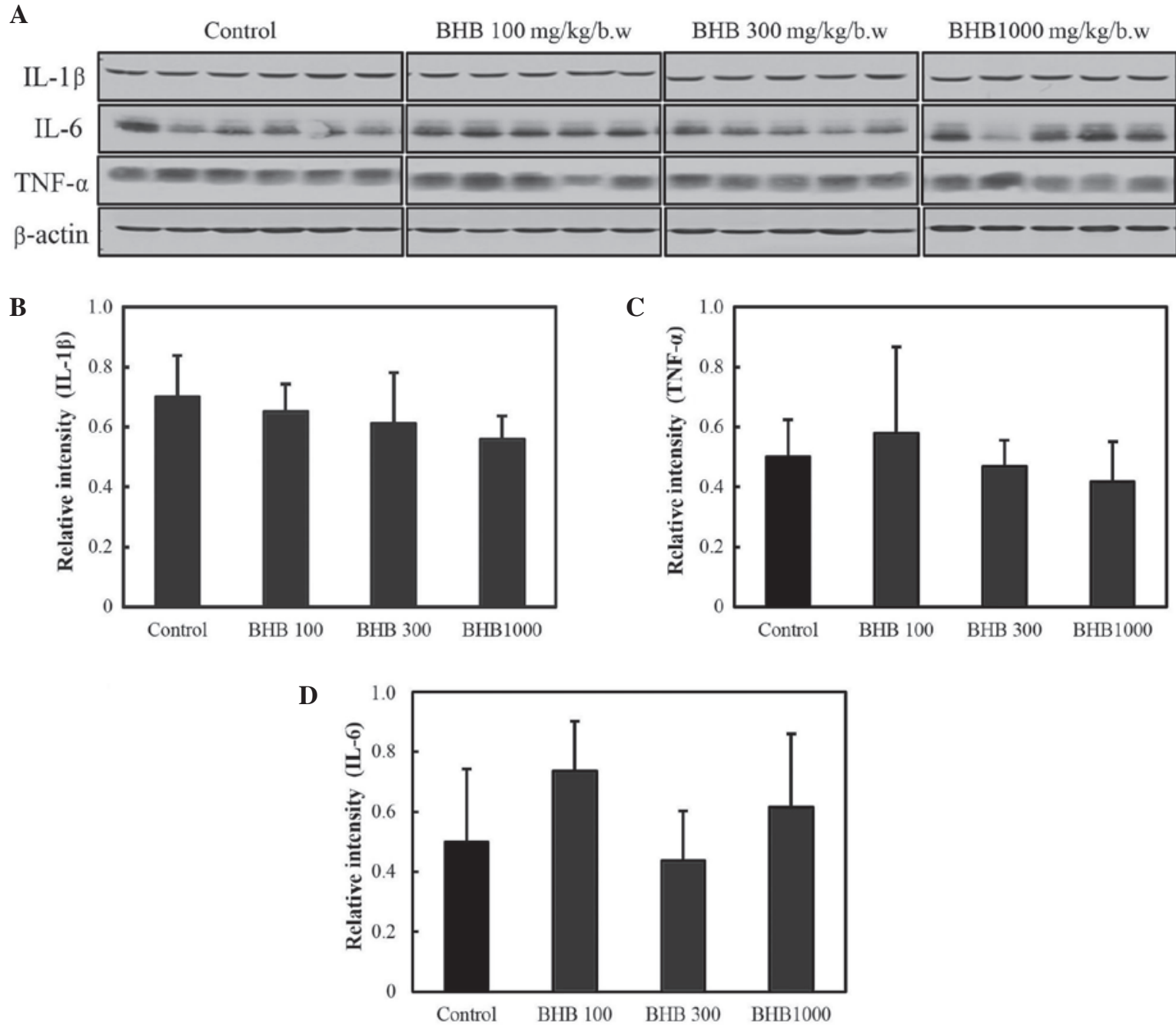

Figure 4. Expression levels of pro-inflammatory cytokines following treatment with BHB, with doses provided in $\mathrm{mg} / \mathrm{kg}$ of body weight. (A) Western blot analysis of pro-inflammatory cytokines; (B) IL-1 $\beta$, (C) TNF- $\alpha$ and (D) IL-6 expression. BHB, benexate hydrochloride betadex; IL, interleukin; TNF- $\alpha$, tumor necrosis factor- $\alpha$. 
A

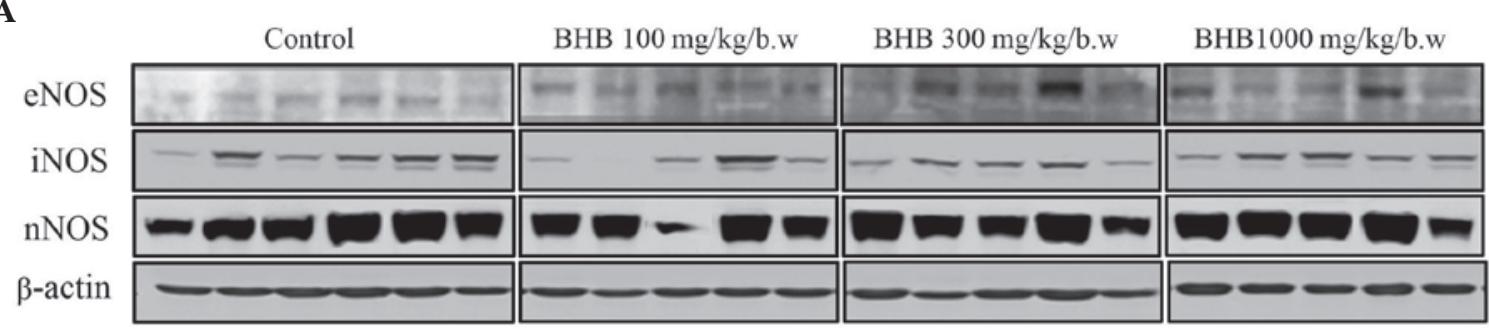

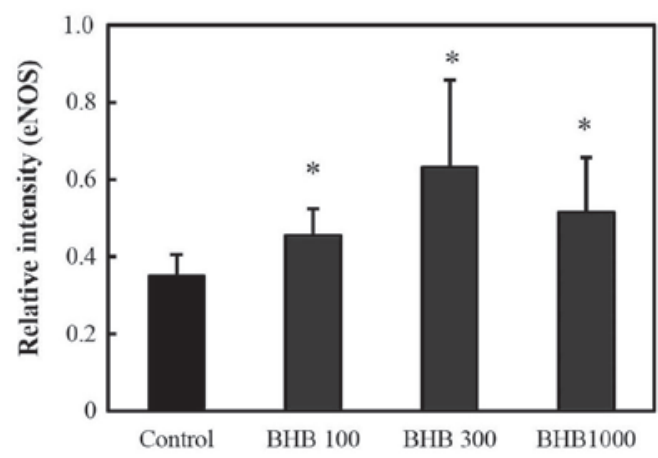

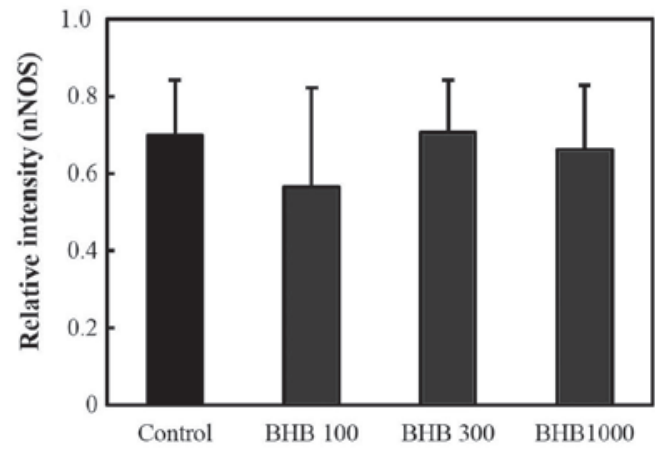

D

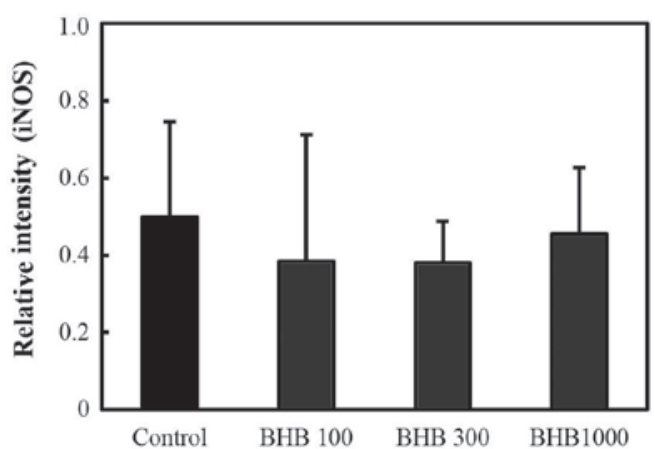

Figure 5. NOS expression following treatment with BHB, with doses provided in $\mathrm{mg} / \mathrm{kg}$. (A) Western blot analysis of NOS expression; (B) eNOS, (C) nNOS and (D) iNOS expression. "P<0.05 vs. the control group. NOS, nitric oxide synthase; BHB, benexate hydrochloride betadex; eNOS, endothelial NOS; nNOS, neuronal NOS; iNOS, inducible NOS.

A

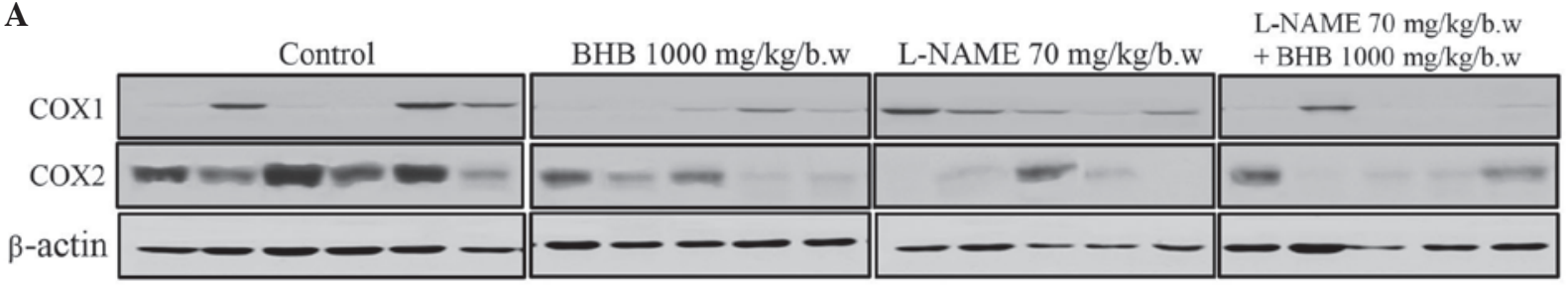

B

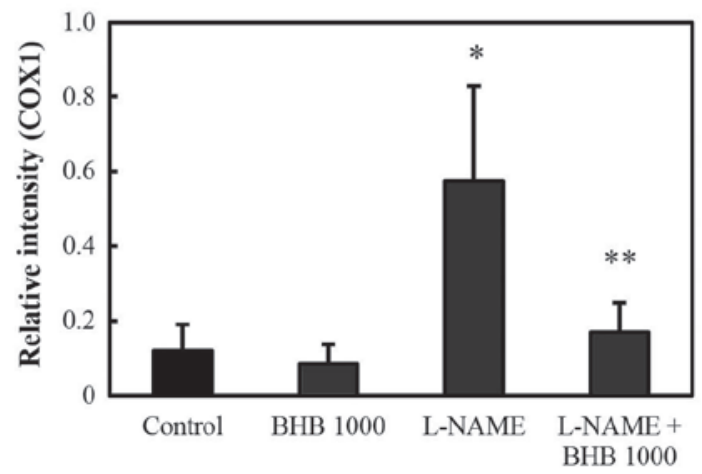

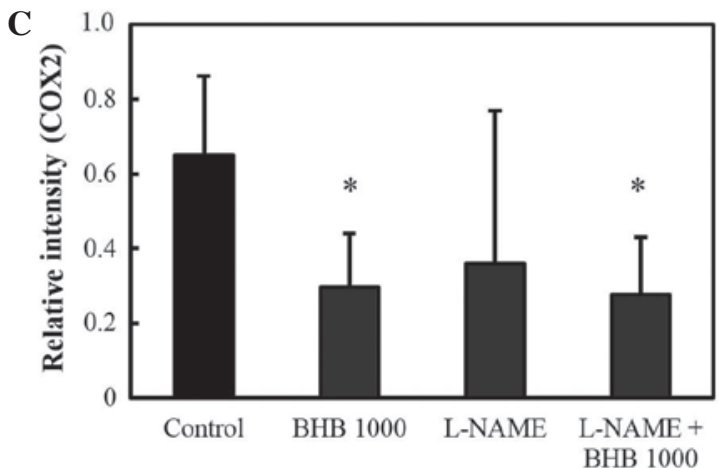

Figure 6.COX expression in rats administered L-NAME with or without BHB.(A) Western blot analysis of COX expression; (B) COX-1 and (C) COX-2 expression . ${ }^{*} \mathrm{P}<0.05$ compared with control; and ${ }^{* *} \mathrm{P}<0.05$ compared with L-NAME treatment only. BHB, benexate hydrochloride betadex; L-NAME, L- $N G$-nitroarginine methyl ester; COX, cyclooxygenase. 


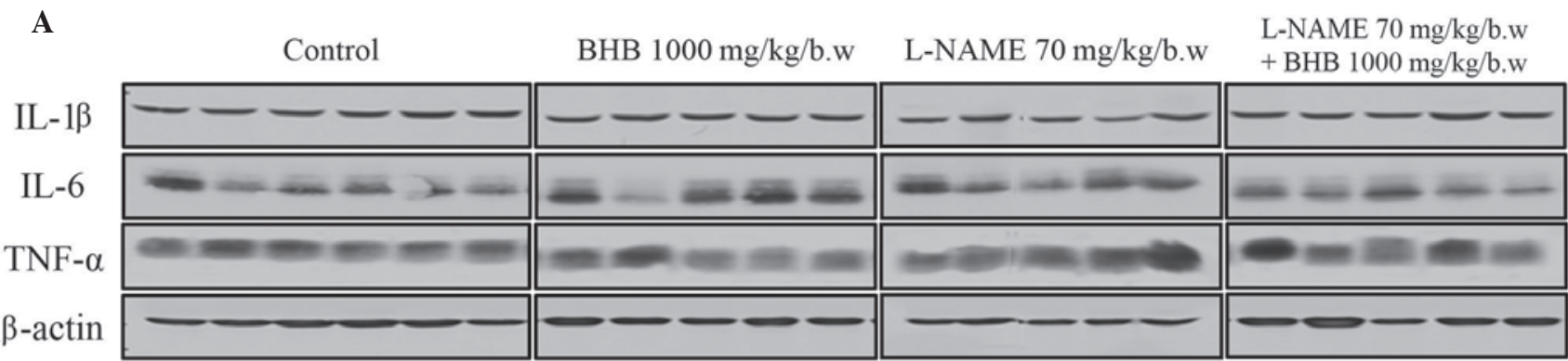

B

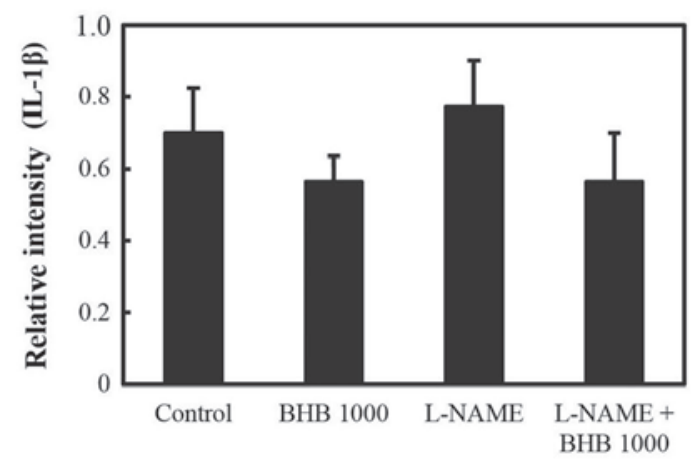

C

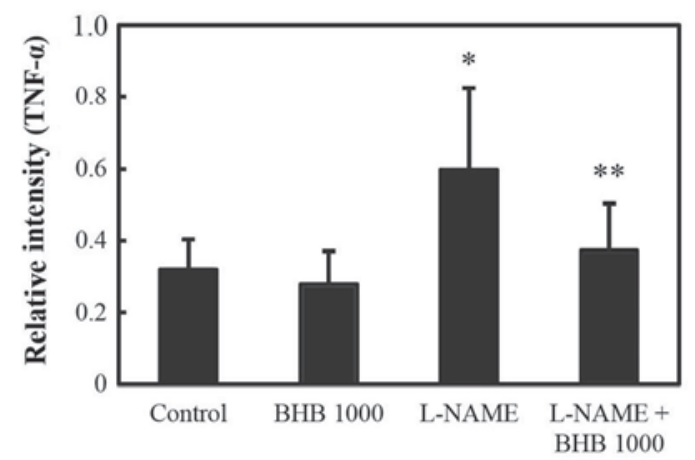

D

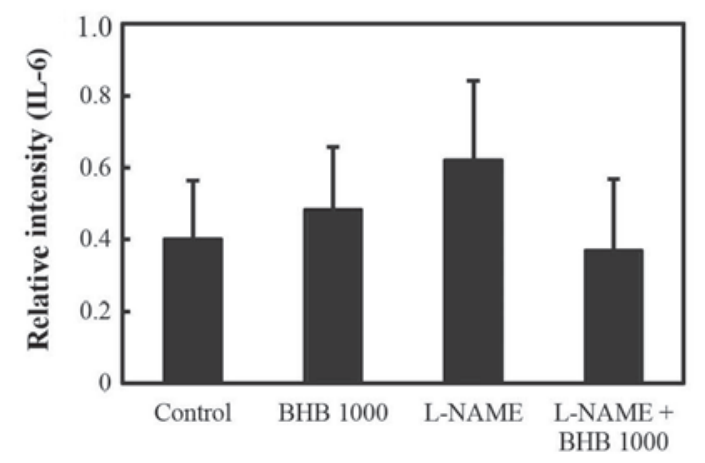

Figure 7. Expression of pro-inflammatory cytokines in rats administered L-NAME with or without BHB. (A) Western blot analysis of pro-inflammatory cytokine expression. Expression of (B) IL-1 $\beta$, (C) IL-6 and (D) TNF- $\alpha$. ${ }^{*} \mathrm{P}<0.05$ vs. the control; and ${ }^{* *} \mathrm{P}<0.05$ vs. L-NAME treatment only. BHB, benexate hydrochloride betadex; L-NAME, L- $N G$-nitroarginine methyl ester; IL, interleukin; TNF- $\alpha$, tumor necrosis factor- $\alpha$.

between those groups that were or were not administered BHB $(\mathrm{P}<0.05$; Fig. 8A and B). The expression levels of nNOS were decreased in the L-NAME group compared with levels in the control group; and compared with the L-NAME group, these levels were significantly increased in the L-NAME + BHB group $(\mathrm{P}<0.05$; Fig. $8 \mathrm{~A}$ and $\mathrm{C})$. By contrast, the expression levels of iNOS were suppressed by BHB + L-NAME co-treatment compared with L-NAME treatment alone $(\mathrm{P}<0.05$; Fig. 8A and D).

\section{Discussion}

The gastric microcirculation has an essential role in healing gastric ulcers. Local vasodilators, including NO, are important in the maintenance of mucosal integrity and in mucosal defense mechanisms (2). Previous studies have reported that increased levels of NO significantly enhance ulcer healing by the maintenance of gastric blood flow; NO also promotes angiogenesis and endothelial cell proliferation and migration (8-10). It has previously been proposed that the anti-ulcer effects of NO result from gastric mucosal NOS activation (3). The calcium-dependent isoforms of NOS, eNOS and nNOS are constitutively expressed in the endothelium of blood vessels and in the brain, respectively. However, expression of iNOS, the calcium-independent isoform, is induced by pro-inflammatory agents $(11,12)$. eNOS is known to promote ulcer healing through angiogenesis, enhancing gastric blood flow and stimulating mucus and bicarbonate secretion (13-15). iNOS is associated with acute and chronic inflammation, and it has previously been reported that changes in iNOS expression and activity levels are correlated with the severity of tissue inflammation (16).

Iwasaki and Matsunaga (17) reported that the vasorelaxant effects of BHB, which were blocked by an NO inhibitor in defective endothelium, were associated with NOS activation. By contrast, Arimoto et al (18) demonstrated that the in vitro activity of NOS was suppressed by BHB. The current study, therefore, hypothesized that a specific NOS may be activated through BHB administration, leading to gastric mucosal healing. In the present study, BHB significantly increased eNOS expression in the rat gastric ulcer model, suggesting that the protective effect of BHB against gastric ulcers may involve an increase in eNOS. iNOS and nNOS were not significantly increased following BHB administration. These 


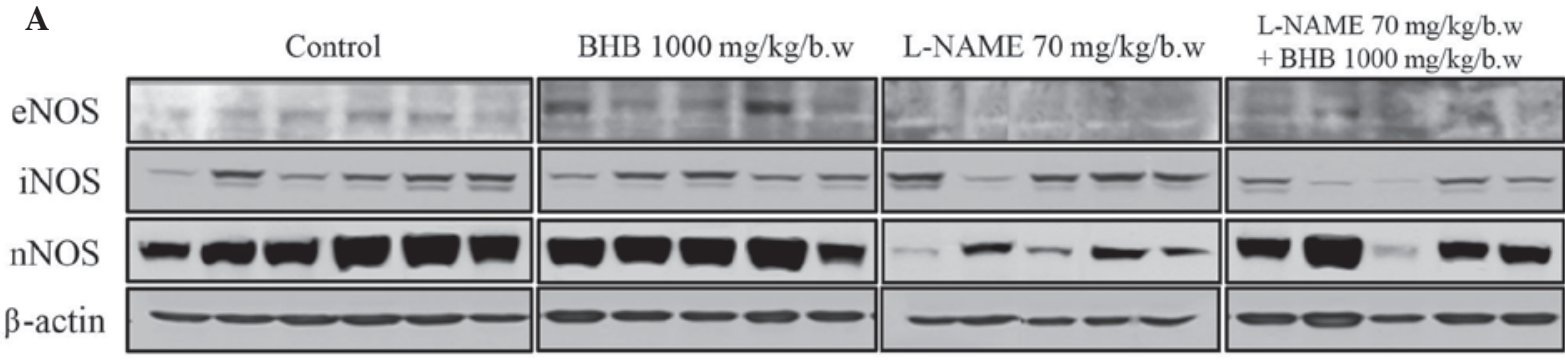

B

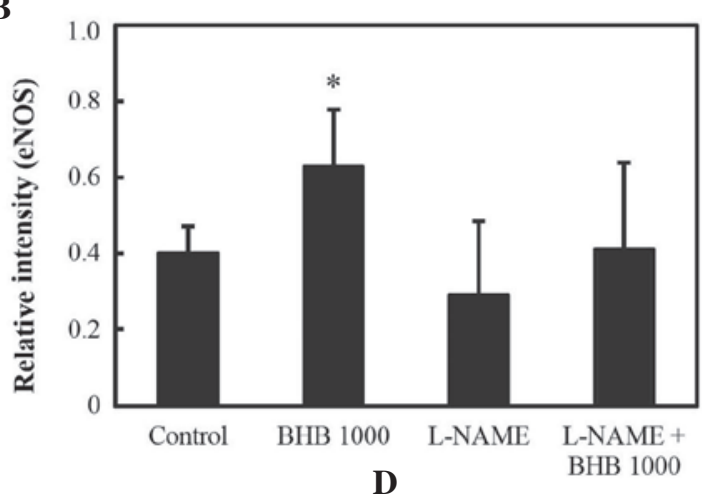

C

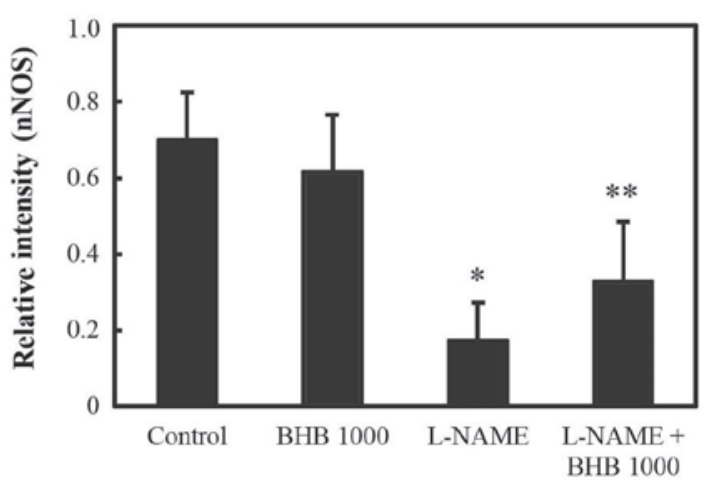

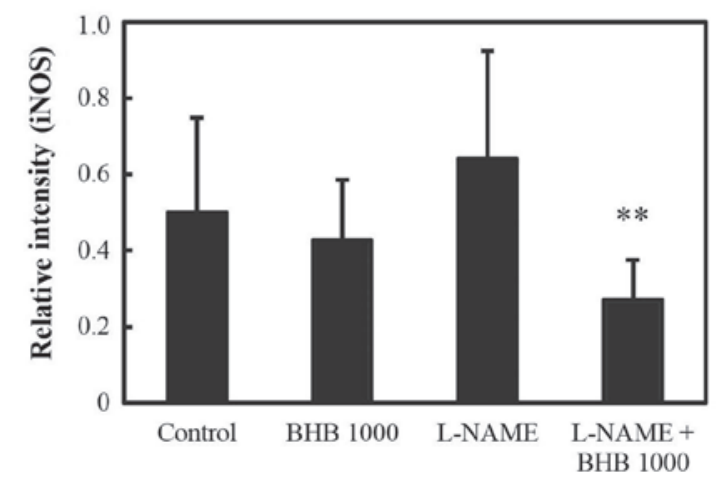

Figure 8. NOS expression in rats administered L-NAME with or without BHB. (A) Western blot analysis of pro-inflammatory cytokine expression. (B) eNOS, (C) nNOS and (D) iNOS expression. ${ }^{*} \mathrm{P}<0.05$ vs. the control; and ${ }^{* *} \mathrm{P}<0.05$ vs. L-NAME treatment only. NOS, nitric oxide synthase; L-NAME, L- $N G$-nitroarginine methyl ester; BHB, benexate hydrochloride betadex; eNOS, endothelial NOS; nNOS, neuronal NOS; iNOS, inducible NOS.

results are partially in agreement with the hypothesis that the anti-ulcer effects of BHB are associated with increased blood flow through the activation of the specific NOS isoform, eNOS. However, BHB did not increase eNOS in the presence of L-NAME, which aggravated the ulcerous lesion. Additional study is therefore required in order to establish the mechanisms of the BHB-induced increased blood flow in gastric ulcers.

An increase in COX-2 expression has previously been demonstrated to be a major contributor to inflammation (10). Previous studies have reported that COX-1 and COX-2 levels are positively correlated with the severity of gastric mucosal damage (19-21). COX-2 mRNA expression is induced during the acute stage of gastric ulceration (22); the early phase of inflammation is primarily mediated by constitutive COX-1 expression, whilst the late and acute phases are mediated by COX-1 and COX-2. COX-2 expression is typically observed 1-2 $\mathrm{h}$ after stimulation (4,23). In the present study, BHB administration at a dose of $1,000 \mathrm{mg} / \mathrm{kg}$ significantly decreased COX-2 levels, which may indicate that the protective effect of $\mathrm{BHB}$ is associated with modulation of COX-2 expression.

Inflammatory cytokines mediate the upregulation of COX-2 expression and inhibit growth factors that are responsible for recovery from mucosal damage $(24,25)$. The current results suggest that $\mathrm{BHB}$ enhances ulcer healing through a reduction of gastric inflammation and of the inflammatory cytokine TNF- $\alpha$; however, no association was observed between BHB and interleukin levels.

There are a number of differences between the mechanism of action of BHB and of other mucoprotective agents, including rebamipide. Rebamipide reportedly suppresses COX-2 expression and NF- $\kappa \mathrm{B}$ activation $(22,26)$. In the present study, however, BHB only decreased COX expression whilst upregulating eNOS. Together, the current results suggest that the anti-ulcer effects of BHB are, in part, associated with decreased COX-2 and pro-inflammatory cytokine levels and with eNOS activation. In conclusion, BHB administration ameliorates acetic acid-induced mucosal injury in a rat model of gastric ulcers by BHB-induced decreases to TNF- $\alpha$ and increases to eNOS expression levels.

\section{Acknowledgements}

This study was supported by the Industrial Core Technology Development Program (grant no. 10060251; Development 
of the diagnostic device for functional dyspepsia based on Korean-Western medicine fusion abdominal diagnosis) and the Basic Science Research Program (grant no. NRF2012R1A2A2A01016829) funded By the Ministry of Trade, Industry and Energy (MI, Korea) and the Ministry of Science, ICT and Future Planning.

\section{References}

1. Hori Y, Odaguchi K, Jyoyama H, Yasui K and Mizui T: Differential effect of benexate hydrochloride betadex on prostaglandin levels in stomach and inflammatory site in rats. Jpn J Pharmacol 72: 183-190, 1996.

2. Pique JM, Whittle BJ and Esplugues JV: The vasodilator role of endogenous nitric oxide in the rat gastric microcirculation. Eur J Pharmacol 174: 293-296, 1989.

3. Ma L and Wallace JL: Endothelial nitric oxide synthase modulates gastric ulcer healing in rats: Am J Physiol Gastrointes Liver Physiol 279: G341-G346, 2000.

4. Brzozowski T, Konturek PC, Sliwowski Z, Pajdo R, Drozdowicz D, Kwiecien S, Burnat G, Konturek SJ and Pawlik WW: Prostaglandin/cyclooxygenase pathway in ghrelin-induced gastroprotection against ischemia-reperfusion injury. J Pharmacol Exp Ther 319: 477-487, 2006.

5. Dembiński A, Warzecha Z, Ceranowicz P, Cieszkowski J, Dembiński M, Ptak-Belowska A, Kuwahara A and Kato I: Administration of obestatin accelerates the healing of chronic gastric ulcers in rats. Med Sci Monit 17: BR196-BR200, 2011.

6. Okabe S, Roth JL and Pfeiffer CJ: A method for experimental, penetrating gastric and duodenal ulcers in rats. Observations on normal healing. Am J Dig Dis 16: 277-284, 1971.

7. Morini G and Grandi D: Methods to measure gastric mucosal lesions in the rat. Curr Protoc Toxicol 21: Unit 21.2, 2010.

8. Kato S, Abe Y,Konishi M, Kuroda N and Takeuchi K: Mechanism of gastric hyperemic response during acid secretion in rats: Relation to nitric oxide, prostaglandins, and sensory neurons. J Clin Gastroenterol 25 (Suppl 1): S48-S55, 1997.

9. Moncada S, Palmer RMJ and Higgs EA: Nitric oxide: Physiology, pathophysiology and pharmacology. Pharmacol Rev 43: 109-142, 1991.

10. Konturek SK and Konturek PC: Role of nitric oxide in the digestive system. Digestion 56: 1-13, 1995.

11. Konturek PC, Brzozowski T, Meixner H, Ptak A, Hahn EG and Konturek SJ: Central and peripheral neural aspects of gastroprotective and ulcer healing effects of lipopolysaccharides. J Physiol Pharmacol 52: 611-623, 2001

12. Brzozowska I, Konturek PC, Brzozowski T, Konturek SJ, Kwiecien S, Pajdo R, Drozdowicz D, Pawlik M, Ptak A and Hahn EG: Role of prostaglandins, nitric oxide, sensory nerves and gastrin in acceleration of ulcer healing by melatonin and its precursor, L-tryptophan. J Pineal Res 32: 149-162, 2002.
13. Guo JS, Cheng CL and Koo MW: Inhibitory effects of Centella asiatica water extract and asiaticoside on inducible nitric oxide synthase during gastric ulcer healing in rats. Planta Med 70: 1150-1154, 2004

14. Cho SO, Lim JW, Kim KH and Kim H: Involvement of Ras and AP-1 in Helicobacter pylori-induced expression of COX-2 and iNOS in gastric epithelial AGS cells. Dig Dis Sci 55: 988-996, 2010.

15. Li Y, Wang WP, Wang HY and Cho $\mathrm{CH}$ : Intragastric administration of heparin enhances gastric ulcer healing through a nitric oxide-dependent mechanism in rats. Eur J Pharmacol 399: 205-214, 2000.

16. McCafferty DM, Mudgett JS, Swain MG and Kubes P: Inducible nitric oxide synthase plays a critical role in resolving intestinal inflammation. Gastroenterology 112: 1022-1027, 1997.

17. Iwasaki $T$ and Matsunaga $K$ : Nitric oxide-associated vasorelaxing effect of an anti-ulcer agent, benexate hydrochloride betadex. Drug Dev Res 36: 13-19, 1995.

18. Arimoto T, Yoshikawa T, Komori Y and Kumagai Y: Inhibition of constitutive nitric oxide synthase by benexate. Life Sci 59: 953-959, 1996.

19. Wallace JL, McKnight W, Reuter BK and Vergnolle N: NSAID-induced gastric damage in rats: Requirement for inhibition of both cyclooxygenase 1 and 2. Gastroenterology 119: 706-714, 2000.

20. Tanaka A, Araki H, Hase S, Komoike Y and Takeuchi K: Up-regulation of COX-2 by inhibition of COX-1 in the rat: A key to NSAID-induced gastric injury. Aliment Pharmacol Ther 16 (Suppl 2): 90-101, 2002.

21. Tanaka A, Araki H, Komoike Y, Hase S and Takeuchi K: Inhibition of both COX-1 and COX-2 is required for development of gastric damage in response to nonsteroidal antiinflammatory drugs. J Physiol Paris 95: 21-27, 2001.

22. Murata H, Yabe Y, Tsuji S, Tsujii M, Fu HY, Asahi K, Eguchi H, Kawano S and Hayashi N: Gastroprotective agent rebamipide induces cyclooxygenease-2 (COX-2) in gastric epithelial cells. Dig Dis Sci 50: S70-S75, 2005.

23. Gudis K and Sakamoto C: The role of cyclooxygenase in gastric mucosal protection. Dig Dis Sci 50 (Suppl 1): S16-S23, 2005.

24. Brzozowska I, Targosz A, Sliwowski Z, Kwiecien S, Drozdowicz D, Pajdo R, Konturek PC, Brzozowski T, Pawlik M, Konturek SJ, et al: Healing of chronic gastric ulcers in diabetic rats treated with native aspirin, nitric oxide (NO)-derivative of aspirin and cyclooxygenase (COX)-2 inhibitor. J Physiol Pharmacol 55: 773-790, 2004.

25. Motilva V, Alarcón de la Lastra C, Bruseghini L, Manuel Herrerias J and Sánchez-Fidalgo S: COX expression and PGE(2) and PGD(2) production in experimental acute and chronic gastric lesions. Int Immunopharmacol 5: 369-379, 2005.

26. Qi Z, Jie L, Haixia C and Xiaoying Z: Effect of rebamipide on quality of peptic ulcer healing in rat. Dig Dis Sci 54: 1876-1883, 2009. 